\title{
Comparing risk models guiding growth factor use in chemotherapy
}

\author{
Chetan Jeurkar, DO; Ho-Man Yeung, MS-IV; Tiffany Pompa, MD; and Michael Styler, MD
}

Drexel University College of Medicine, Division of Hematology/Oncology, Philadelphia, Pennsylvania

Background The National Comprehensive Cancer Network (NCCN) and the American Society of Clinical Oncology (ASCO)
have guidelines for using colony-stimulating factors (CSF) for chemotherapy-induced neutropenia (CIN). Both groups recommend
CSF if the chemotherapy has a risk for febrile neutropenia of more than $20 \%$. The guidelines are less definitive if the risk is inter-
mediate (10\%-20\%). Two risk models developed by Hosmer and Bozcuk and their respective colleagues may provide guidance
regarding CSF decision making in this intermediate risk population. Objective To examine whether risk models developed by Hosmer and Bozcuk had adjunct value to the NCCN and ASCO guidelines when applied to patients with lung cancer who were receiving intermediate-risk chemotherapy.

Methods Male and female patients aged 18-75 years with a diagnosis of any stage lung cancer, small or non-small cell, who required and received their initial chemotherapy at Drexel University in Philadelphia were included in this study. Patients who received growth factor before their chemotherapy were excluded. The Hosmer and Bozcuk calculators for febrile neutropenia risk and the NCCN and ASCO guidelines for using CSF for CIN were applied to this group of patients.

Results 43 patients were included in the study. The Hosmer and Bozcuk calculators and NCCN and ASCO guidelines recommended giving CSF to $26,22,25$, and 38 patients, respectively. The sensitivities for detecting severe CIN were $89 \%$, $78 \%, 67 \%$, and $97 \%$, and the specificities were $44 \%, 56 \%, 45 \%$, and $14 \%$, respectively.

Limitations Small cohort size; data were limited in scope.

Conclusions In lung cancer patients receiving intermediate-risk chemotherapy, the Hosmer calculator had the best combination of sensitivity, specificity, and ease of use. The NCCN guidelines were less sensitive, whereas the ASCO guidelines were the least specific. Based on these findings, we recommend using the Hosmer calculator because it lends to accurate but judicious use of CSF.

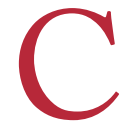
hemotherapy-induced neutropenia (CIN) and its corollary febrile neutropenia (FN) are well recognized, and they are serious consequences of many agents used in the treatment of malignancy. FN in particular has been associated with a considerable risk of morbidity and mortality, namely sepsis with multiorgan failure and eventual death. ${ }^{1}$ The mainstay of prophylaxis for patients who are deemed to be at high risk for CIN and FN is colony-stimulating factors (CSF). These agents have been shown to significantly decrease FN-related mortality, and therefore their use is potentially lifesaving. ${ }^{2}$ However, CSF are not cheap, with the cost of peg-filgrastim as much as US $\$ 6195.99$ per cycle of chemotherapy. ${ }^{3}$ Therefore, not only do FN and CIN pose significant risk to patients, they also carry a high burden of cost to the patient and health care system both in treatment and prophylaxis. ${ }^{4}$ As such, it is prudent for oncologists to accurately identify high-risk patients and judiciously use CSF in an evidence-based manner.

However, this has proven to be difficult because of the extent of variability between patients and the heterogeneity of the various risk models in the literature. Currently, there are 2 widely used guidelines, 1 developed by the National Comprehensive Cancer Network (NCCN) and another by the American Society of Clinical Oncology (ASCO). Both guidelines suggest the use of prophylactic $\mathrm{CSF}$ if the chemotherapy regimen has an FN risk of more than $20 \%$ (high risk). If the chemotherapy is deemed to be of intermediate risk (10\%-20\% FN risk), then patient-specific factors need to be considered. 5,6

In lung cancer, the NCCN lists only topotecan for small cell carcinomas as being high risk for $\mathrm{FN}$, and therefore it is the only regimen that would warrant definitive use of prophylactic CSF. ${ }^{5}$ The most recent ASCO guidelines do not list chemotherapy regimens that are high risk for FN. ${ }^{6}$ For intermediate-risk regimens, the NCCN states that CSF prophylaxis should be considered if the patient has had previous chemotherapy or radiation therapy, persistent neutropenia, bone marrow involvement by

Accepted for publication November 2, 2018. Correspondence: Chetan Jeurkar, DO; jeurkar2@gmail.com. Disclosures: The authors report no disclosures or conflicts of interest. JCSO 2018;16(5):e256-e259. (C2018 Frontline Medical Communications. doi: https://doi.org/10.12788/jcso.0429 
tumor, recent surgery or open wounds, liver dysfunction (total bilirubin, $>2.0 \mathrm{mg} / \mathrm{dL}$ ), or renal dysfunction (creatinine clearance, $<50 \mathrm{~mL} / \mathrm{min}$ ), or is older than 65 years. ${ }^{5}$

ASCO guidelines state that in intermediate-risk chemotherapy regimens, the following factors are to be considered: age $>65$ years, advanced disease, previous chemotherapy or radiation therapy, pre-existing neutropenia or marrow involvement by tumor, infection, open wounds or recent surgery, poor performance status or nutritional status, poor renal function, liver dysfunction (most notably bilirubin elevation), cardiovascular disease, multiple comorbid conditions, and HIV infection. However, in the ASCO guidelines, there is no suggestion as to whether CSF should be administered if patients have one of these risk factors, only to "consider these factors when estimating patients' overall risk of febrile neutropenia." 6

There is some uncertainty with the NCCN and ASCO guidelines as to whether prophylactic CSF should be given to these intermediate-risk patients. There are suggestions but no definitive guidelines. In our study, we looked at lung cancer patients treated with intermediate-risk chemotherapy regimens and applied 2 different risk models created by Hosmer $^{7}$ and Bozcuk ${ }^{8}$ and their respective colleagues (Hosmer and Bozcuk hereinafter). Our goal was to assess the efficacy differences between the 2 risk models and to compare their outcomes and recommendations with the NCCN and ASCO guidelines. This was done to showcase the tools available to a clinical oncologist who must decide whether to prescribe prophylactic CSF in these more challenging clinical situations.

\section{Methods \\ Study population}

This was a cross-sectional, retrospective study looking at male and female patients aged 18 to 75 years who were treated in the hematology-oncology offices of Drexel University in Philadelphia, Pennsylvania, from 2005 through 2016, who had a diagnosis of lung cancer and were, at some point during their disease, treated with chemotherapy. By using ICD-10 codes for any type of lung cancer, we identified 242 patients. Of those, 106 patients were excluded because they had never received chemotherapy, 16 were excluded either because of miscoding of the type of cancer or because they never actually had cancer, and 61 were excluded either because chemotherapy had not been delivered at our institution or because there were insufficient data to apply the 2 risk models. Of the remaining 59 patients, 16 were excluded because they had received prophylactic CSF with their first cycle of chemotherapy, leaving a total of 43 patients to whom the various risk models and guidelines could be applied (Table 1). If any of the 43 patients were found to be neutropenic, they were given growth factor shortly thereafter.

\begin{tabular}{|c|c|}
\hline Characteristic & n (\%) \\
\hline Age $>60 y$ & $27(62.7)$ \\
\hline Male & $16(37.2)$ \\
\hline \multicolumn{2}{|l|}{ Lung cancer type } \\
\hline Adenocarinoma & $26(60.4)$ \\
\hline Squamous cell & 12 (27.9) \\
\hline Small cell & $3(6.9)$ \\
\hline Undifferentiated & $2(4.6)$ \\
\hline \multicolumn{2}{|l|}{$\begin{array}{l}\text { Stage at time of } \\
\text { chemotherapy }\end{array}$} \\
\hline I & $5(11.6)$ \\
\hline ॥ & 5 (1 11.7$)$ \\
\hline III & $19(44.1)$ \\
\hline IV & $14(32.5)$ \\
\hline \multicolumn{2}{|c|}{ Chemotherapy regimen } \\
\hline Platinum doublet & $32(74.4)$ \\
\hline Monotherapy & $11(25.6)$ \\
\hline
\end{tabular}

Chemotherapy for these 43 patients consisted of either a platinum doublet (cisplatin or carboplatin with either etoposide, pemetrexed, gemcitabine, or paclitaxel) or monotherapy with either paclitaxel, abraxane, navelbine, or pemetrexed. Of the 43 patients, 32 had platinum-based doublets, and 11 had monotherapy with one of the listed agents (Table 1).

Formal patient consent was not required because this was a retrospective study.

\section{Defining CIN and FN}

Neutropenia was defined as an absolute neutrophil count (ANC) of less than 1500 neutrophils per microliter. The levels of neutropenia were defined as mild (ANC, 10001500 neutrophils $/ \mu \mathrm{L}$ ), moderate (ANC, 500-1000 neutrophils $/ \mu \mathrm{L}$ ), and severe (ANC, $<500$ neutrophils $/ \mu \mathrm{L})$. The NCCN guidelines define $\mathrm{FN}$ as a single temperature of $>38.3^{\circ} \mathrm{C}$ orally or $>38.0^{\circ} \mathrm{C}$ over 1 hour, with an associated ANC of $<500$ or $<1000$ with a predicted decline to $<500$ over the next 48 hours. $^{5}$

\section{Risk models}

It should be noted that the Hosmer and Bozcuk calculators were powered to detect occurrence of FN. ${ }^{7,8}$ However, we also applied them for the risk of any CIN. In scoring for the Hosmer calculator, points are given to each risk factor and are added together to give a final risk score. This risk score correlates to a percentage of predicted FN. The score for the Hosmer calculator is from minus 18 to plus 19, in 
TABLE 2 Sensitivity and specificity values for the Hosmer and Bozcuk risk models and the NCCN and ASCO guidelines for FN risk

\begin{tabular}{lcccc}
$\begin{array}{l}\text { Risk model/ } \\
\text { guideline }\end{array}$ & $\begin{array}{c}\text { Severe CIN } \\
\text { sensitivity }\end{array}$ & $\begin{array}{c}\text { Severe CIN patients not } \\
\text { recommended CSF }\end{array}$ & FN sensitivity & $\begin{array}{c}\text { CIN and FN } \\
\text { specificity }\end{array}$ \\
\hline Hosmer & 89 & 1 & 100 & 44 \\
\hline Bozcuk & 78 & 2 & 75 & 56 \\
NCCN & 67 & 3 & 50 & 45 \\
ASCO & 97 & 1 & 100 & 14
\end{tabular}

ASCO, American Society of Clinical Oncology; CIN, chemotherapy-induced neutropenia; CSF, colony-stimulating factors; FN, febrile neutropenia; NCCN, National Comprehensive Cancer Network

which a score of 13 or higher correlates to a $15 \%$ predicted risk of $\mathrm{FN}$, and a score of 0 or less correlates to a $1.6 \%$ risk of FN. ${ }^{7}$ For the Bozcuk calculator, a nomogram is used to calculate risk. Individual points are given to each risk factor and are then summed to give a total that correlates to a risk of FN. The score range for the Bozcuk calculator is 0 to 300 , with a score of greater than 190 correlating to a greater than $90 \%$ risk of $\mathrm{FN}$, and a score of 0 correlating to a $0 \%$ predicted risk of $\mathrm{FN} .^{8}$

For sensitivity and specificity threshold values, Hosmer reported using a risk score of 10 or above as being a reasonable value for the use of prophylactic CSF. They reported this score would predict an FN risk of about 10\%, sensitivity of $24 \%$, and specificity of $93 \%$ in detecting FN. ${ }^{7}$ Bozcuk reported that using 110 as a cutoff value would correlate to about a 50\% FN risk, sensitivity of $100 \%$, and specificity of 49\%. However, they did not suggest that value be applied as a threshold for the use of prophylactic CSF as Hosmer did. ${ }^{8}$ Despite that, we used the thresholds of 10 and 110 for sensitivity and specificity analyses.

Regarding the current cycle of chemotherapy, the Hosmer calculator looked only at the first cycle, whereas the Bozcuk calculator looked at any cycle of chemotherapy. ${ }^{7,8}$ In our study, we used the cycle correlating to the lowest ANC nadir the patient achieved. For example, if a patient achieved a nadir of 1,000 in cycle 1 but 200 in cycle 2 , then we used the cycle 2 data to complete the calculators.

With respect to the NCCN and ASCO guidelines, we evaluated our cohort of 43 patients for the risk factors listed in the respective guidelines. If a patient had 1 or more of the risk factors, they were deemed to be high risk and therefore were recommended to receive CSF.

\section{Results}

\section{General data}

Of the 43 patients studied, 21 developed some level of CIN. Nine patients developed severe CIN, 4 developed moderate CIN, and 8 developed mild CIN. Of the severely neutropenic patients, 4 developed FN. None of the 16 patients who received prophylactic CSF developed $\mathrm{FN}$, although 2 developed severe neutropenia despite CSF administra- tion. Nadirs of ANC were seen on average during cycle 3 of chemotherapy. In all, 15 of the 43 patients achieved lowest ANC nadir during cycle 1 .

\section{Risk models}

The Bozcuk calculator. A total of 22 patients had risk scores above the calculator's threshold value of 110 . Of those 22 patients, 7 developed severe CIN, 5 developed either mild or moderate CIN, and 3 developed FN. Of the remaining 21 patients who had risk scores of below 110, 2 developed severe CIN, 7 developed mild or moderate CIN, and 1 developed FN. Sensitivity and specificity values are shown in Table 2.

The Hosmer calculator. A total of 26 patients had risk scores above the calculator's threshold value of 10 . Of those 26 patients, 8 developed severe CIN, 4 developed either mild or moderate CIN, and 4 developed FN. Of the remaining 17 patients who had risk scores of less than 10,1 developed severe CIN, 8 developed mild or moderate CIN, and none developed FN. Sensitivity and specificity values are listed in Table 2.

\section{Current guidelines}

NCCN guidelines. If one were to use the NCCN guidelines on our cohort of 43 patients, 25 would have been recommended to receive prophylactic CSF. Of those 25, 6 developed severe CIN (2 with FN), 2 moderate CIN, and 5 mild CIN. Of the 18 patients who would not have been recommended to receive CSF, 3 developed severe CIN (with 2 FN), 2 moderate CIN, and 3 mild CIN. Sensitivity and specificity values are listed in Table 2 .

ASCO guidelines. Using the ASCO guidelines on our cohort of 43 patients, 38 had 1 or more of the high-risk features, and, therefore, CSF would have been considered for them. Of those 38 patients, 8 developed severe CIN (4 with FN), 4 developed moderate CIN, and 7 developed mild CIN. Of the 5 patients who would not have received CSF, 1 developed severe CIN and 1 mild CIN. Sensitivity and specificity values are listed in Table 2 . 


\section{Discussion}

In our study, we looked at 2 CIN risk models and compared them with the current NCCN and ASCO guidelines. The models were created to predict risk of $\mathrm{FN}$, but we also looked at their predictive value for any level of CIN. To this end, we found that the Hosmer and Bozcuk calculators both were acceptable for predicting risk of severe CIN and FN. Because of the small number of patients in this study, differences in sensitivities and specificities cannot be quantitatively compared. Nevertheless, qualitatively, it can be said that both calculators were accurate in assigning high-risk scores to patients who developed severe CIN or FN. However, both calculators had many patients with high-risk scores who never developed CIN.

When comparing the 2 risk models with the NCCN and ASCO guidelines, the ASCO guidelines tended to be more liberal in their consideration of CSF use, whereas the NCCN guidelines tended to be more conservative and more similar to the 2 risk models we tested. The NCCN guidelines suggested not giving prophylactic CSF to 2 of our patients who developed FN and to not give CSF to an additional patient who developed severe CIN. The ASCO guidelines suggested considering using CSF for most of our patients, with only 5 patients not to be considered for CSF administration.

The differences in efficacy between the current guidelines and the 2 risk models may be indicative of the fact that the risk models are more accurate in assigning risk in older patients who are clinically more complicated. In our patients, the chemotherapies used were all considered to be intermediate risk, so patient-specific factors were used to guide the administration of CSF. However, because many our patients had at least 1 of the risk factors listed by the $\mathrm{NCCN}$ or ASCO, they were automatically deemed to be high risk and to receive prophylactic CSF.

Consequently, the Hosmer and Bozcuk calculators may be of greatest utility in more clinically complicated patients and those who have more comorbidities. The best approach may be a combination of either the NCCN or ASCO guidelines with 1 of the calculators, in our opinion the Hosmer system, for these complicated patients. Likely, the 2 risk models would not be as useful for chemotherapies deemed to have a high risk for $\mathrm{FN}$ because, in those situations, the efficacy and benefit of prophylactic CSF are clear. ${ }^{9}$ Rather, their use could be beneficial in the grayer areas in which the risk is intermediate and decision-making is more difficult.

\section{Limitations}

There were several limitations in our study. First, the size of the cohort was small, and, therefore, the data that we gathered was limited in its scope. However, the goal of this study was to help provide guidance to oncologists in realworld settings about the validity and use of the available risk calculators. A further study should compare the calculators and guidelines in a much larger cohort to see if present results still hold true.

The second possible limitation of the study was our application of the Hosmer calculator because our patient population did not fit the criteria for inclusion in their original study. Hosmer had included only the first cycle of chemotherapy, whereas we included all cycles of chemotherapy. However, despite that, the calculator still performed well and could predict severe CIN and FN even with later cycles of chemotherapy. Therefore, we suggest using this calculator in any cycle of chemotherapy rather than just the first. This would expand its scope and utility in clinical practice.

\section{Conclusions}

This article provides oncologists with a comparison of 2 CIN risk models with the currently available NCCN and ASCO guidelines for use in patients with lung cancer. We prefer the Hosmer calculator over the Bozcuk calculator because of its simplicity of use and the accuracy of results. We anticipate that it may be useful and practical as an adjunct tool to the NCCN or ASCO guidelines in patients receiving intermediate-risk chemotherapy regimens. Larger studies combining the calculators and determining accuracy need to be completed to prove this hypothesis.

\section{References}

1. Kuderer NM, Dale DC, Crawford J, Cosler LE, Lyman GH. Mortality, morbidity, and cost associated with febrile neutropenia in adult cancer patients. Cancer. 2006;106(10):2258-2266.

2. Kuderer NM, Dale DC, Crawford J, Lyman GH. Impact of primary prophylaxis with granulocyte colony-stimulating factor on febrile neutropenia and mortality in adult cancer patients receiving chemotherapy: a systematic review. J Clin Oncol. 2007;25(21):3158-3167.

3. Good Rx, Inc. Peg-filgrastim. https://www.goodrx.com/neulasta. Accessed September 2018.

4. Schilling MB, Parks C, Deeter RG. Costs and outcomes associated with hospitalized cancer patients with neutropenic complications: a retrospective study. Exp Ther Med. 2011;2(5):859-866.

5. National Comprehensive Cancer Network. Myeloid growth factors. In: NCCN Clinical Practice Guidelines in Oncology. Plymouth Meeting, PA: National Comprehensive Cancer Network; 2018.

6. Smith T, Bohlke K, Lyman GH, et al. Recommendations for the use

of WBC growth factors: American society of clinical oncology clinical practice guideline update. J Clin Oncol. 2015;33(28):3199-3212.

7. Hosmer W, Malin J, Wong M. Development and validation of a prediction model for the risk of developing febrile neutropenia in the first cycle of chemotherapy among elderly patients with breast, lung, colorectal, and prostate cancer. Support Care Cancer. 2011;19(3):333-341.

8. Bozcuk H, Yıldız M, Artaç M, et al. A prospectively validated nomogram for predicting the risk of chemotherapy-induced febrile neutropenia: a multicenter study. Support Care Cancer. 2015;23(6):1759-1767.

9. Vogel CL, Wojtukiewicz MZ, Carroll RR, et al. First and subsequent cycle use of pegfilgrastim prevents febrile neutropenia in patients with breast cancer: a multicenter, double-blind, placebo-controlled phase III study. J Clin Oncol. 2005;23(6):1178-1184. 